\title{
Minimal multi-convex projections
}

\author{
by \\ Grzegorz Lewicki (Kraków) and Michael Prophet (Cedar Falls, IA)
}

\begin{abstract}
We say that a function from $X=C^{L}[0,1]$ is $k$-convex (for $k \leq L$ ) if its $k$ th derivative is nonnegative. Let $P$ denote a projection from $X$ onto $V=\Pi_{n} \subset X$, where $\Pi_{n}$ denotes the space of algebraic polynomials of degree less than or equal to $n$. If we want $P$ to leave invariant the cone of $k$-convex functions $(k \leq n)$, we find that such a demand is impossible to fulfill for nearly every $k$. Indeed, only for $k=n-1$ and $k=n$ does such a projection exist. So let us consider instead a more general "shape" to preserve. Let $\boldsymbol{\sigma}=\left(\sigma_{0}, \sigma_{1}, \ldots, \sigma_{n}\right)$ be an $(n+1)$-tuple with $\sigma_{i} \in\{0,1\}$; we say $f \in X$ is multi-convex if $f^{(i)} \geq 0$ for $i$ such that $\sigma_{i}=1$. We characterize those $\sigma$ for which there exists a projection onto $V$ preserving the multi-convex shape. For those shapes able to be preserved via a projection, we construct (in all but one case) a minimal norm multi-convex preserving projection. Out of necessity, we include some results concerning the geometrical structure of $C^{L}[0,1]$.
\end{abstract}

1. Introduction. When $X$ is a Banach space and $V \subset X$ a subspace, we denote by $\mathcal{P}(X, V)$ the set of all projections from $X$ onto $V$; in the cases where there is no ambiguity, we will simply write $\mathcal{P}$. We say that a projection $P_{0}$ is minimal if $\left\|P_{0}\right\| \leq\|P\|$ for all $P \in \mathcal{P}(X, V)$.

There exist a large number of papers concerning minimal projections. The problems considered are mainly existence ([15], [18]), uniqueness ([14], [16], [27], [39], [40]), characterization of one-complemented subspaces ([1], $[2],[29],[36],[37],[19])$ concrete formulas for minimal projections ([3]-[7], [13], [15], [23], [24], [26], [35], [41]), estimates of the relative projection constants ([5], [17], [21], [25], [33], [38], [42]), construction of spaces with large relative projection constants ([4], [5], [20], [22]). For basic information concerning this topic the reader is referred to [32].

While a minimal projection will, in general, provide good approximations, it may fail to preserve particular properties of elements, as illustrated below. We are therefore motivated to look for projections which leave invari-

2000 Mathematics Subject Classification: 46B28, 47A58, 41A65.

Key words and phrases: multi-convex function, minimal projection, shape-preserving projection.

The first author is supported by KBN Grant 1 P03A 01026. 
ant (or preserve) a particular functional characteristic (or "shape"). These characteristics are often described using cones.

More precisely, a cone in $X$ is a convex set closed under nonnegative scalar multiplication. Assuming $\mathcal{P} \neq \emptyset$, we may fix a cone $S \subset X$ and ask if any element from $\mathcal{P}$ leaves $S$ invariant; i.e., let

$$
\mathcal{P}_{S}=\mathcal{P}_{S}(X, V)=\{P \in \mathcal{P} \mid P S \subset S\}
$$

and determine if $\mathcal{P}_{S} \neq \emptyset$. When $P \in \mathcal{P}_{S}$ we say $P$ is shape-preserving (in the sense of $S$ ). Some basic results on the existence of shape-preserving projections can be found in [10], [31], [12] and [34]. Not surprisingly, for given $X, V$ and $S$, the problem of determining if $\mathcal{P}_{S} \neq \emptyset$ is nontrivial in general.

In this paper we first characterize, for a large collection of $X, V$ and $S$, when $\mathcal{P}_{S} \neq \emptyset$; then, for each setting in which $\mathcal{P}_{S} \neq \emptyset$, we calculate $\inf _{P \in \mathcal{P}_{S}}\|P\|$. Moreover we construct a minimal shape-preserving projection.

Specifically, for positive integer $L$ let $X$ denote the $L$-times continuously differentiable functions on $[0,1], C^{L}[0,1]$, normed by

$$
\|f\|_{L}=\max _{i=0, \ldots, L}\left\{\left\|f^{(i)}\right\|_{\infty}\right\} .
$$

In this case we simply write $X=\left(C^{L}[0,1],\|\cdot\|_{L}\right)$. We denote by $X^{*}$ the dual space of $X$. In this setting, note that $\delta_{t}^{k}$, the $k$ th derivative evaluation at $t$, belongs to the unit sphere of $X^{*}$ for $k=0, \ldots, L$ and $t \in[0,1]$. For fixed $k$, consider the cone $S \subset X$ of all $f \in X$ with nonnegative $k$ th derivative on $[0,1]$. We refer to this set as the cone of $k$-convex functions. With $V=\Pi_{n}$, the $n$th degree algebraic polynomials, it was shown in [11] that

$$
\mathcal{P}_{S} \neq \emptyset \Leftrightarrow k \geq n-1 \text {. }
$$

For example, for $X=\left(C^{1}[0,1],\|\cdot\|_{1}\right)$ and $k=1$ we see that there is no monotonicity-preserving (1-convex preserving) projection from $X$ onto $V=\Pi_{3}$. There is however a projection preserving convexity (or 2-convexity) onto $V$. Moreover, Theorem 4.2 in [11] constructs a minimal norm element of $\mathcal{P}_{S}$ for $k=n-1$ (with norm $3 / 2$ for every $n$ ) using techniques from minimal projection theory found in [7].

As we will see in Section 3, the existence of a projection onto $\Pi_{n}$ preserving $k$-convexity can be determined via geometric considerations; in the case $k=n$ or $k=n-1$, this geometric approach reduces (respectively) to a 1-dimensional or 2-dimensional problem and is relatively easy to solve. That is, the geometric approach quickly reveals the result in (1).

We now look to generalize $k$-convexity. Using notation similar to that of [30], for a fixed positive integer $n$ let $\boldsymbol{\sigma}=\left(\sigma_{0}, \sigma_{1}, \ldots, \sigma_{n}\right)$ be an $(n+1)$-tuple with $\sigma_{i} \in\{0,1\}$; let $M=\max _{\sigma_{i}=1} i$. With $X=C^{L}[a, b](L \geq M)$, define

$$
S_{\boldsymbol{\sigma}}:=\left\{f \in X \mid \sigma_{i} f^{(i)} \geq 0, i=0, \ldots, n\right\} .
$$


We say $f \in X$ is multi-convex if $f$ belongs to the cone $S_{\boldsymbol{\sigma}}$. In this paper we fix $V=\Pi_{n}$ and consider projections from $X$ onto $V$ leaving invariant a cone of multi-convex functions, so-called multi-convex projections. We denote this set of projections by $\mathcal{P}_{S_{\boldsymbol{\sigma}}}$ and look to construct minimal norm elements from this set.

This paper is organized into five sections. Following these introductory remarks, the main content of this paper is described in Section 2. There we characterize those $\boldsymbol{\sigma}$ for which $\mathcal{P}_{S \boldsymbol{\sigma}} \neq \emptyset$, where $\mathcal{P}_{S \boldsymbol{\sigma}} \subset X=\left(C^{L}[0,1],\|\cdot\|_{L}\right)$. Furthermore, we develop an iterative, norm-preserving construction of multiconvex projections from $X$ onto $(n+1)$-dimensional subspaces $V$, where the iteration is with respect to $n$. This construction yields minimal norm multiconvex projections in the case $V=\Pi_{n}$. Sections 3 and 5 provide proofs of the results of Section 2. The proofs in Section 5 require basic, nontrivial facts about the unit ball of $X=\left(C^{L}[0,1],\|\cdot\|_{L}\right)$. For completeness, we prove the needed results in Section 4 (indeed we found no single source which described the geometry of this ball and thus hope that Section 4 may be of independent utility to others).

As a summary of notation used in the following, the dual space of Banach space $X$ is denoted by $X^{*}$; we denote by $B(X)$ and $S(X)$, respectively, the unit ball of $X$ and the unit sphere of $X$. For a convex set $K \subset X$, we denote by $\operatorname{ext}(K)$ the set of extreme points of $K$. The convex hull of a subset $A \subset X$ is denoted by co $A$ while the convex cone generated by $A$ is cone $(A)=\{\varrho a \mid \varrho \in[0, \infty)$ and $a \in A\}$.

2. Main results. Let $L$ and $n$ denote positive integers such that $L \geq$ $n-1$ (the reason for this inequality will be made clear). Let $\boldsymbol{\sigma}=\left(\sigma_{0}, \sigma_{1}\right.$, $\left.\ldots, \sigma_{n}\right)$ with $\sigma_{i} \in\{0,1\}$; let

$$
M=\max _{\sigma_{i}=1} i \quad \text { and } \quad m=\min _{\sigma_{i}=1} i .
$$

We say that $\boldsymbol{\sigma}$ is 1 -connected if whenever $\sigma_{i}=\sigma_{j}=1$ for $i<j$, we have $\sigma_{k}=1$ for all $k=i, i+1, \ldots, j$.

Theorem 2.1. Let $X=\left(C^{L}[0,1],\|\cdot\|_{L}\right)$. Then $\mathcal{P}_{S_{\boldsymbol{\sigma}}}\left(X, \Pi_{n}\right) \neq \emptyset$ iff $M \geq n-1$ and $\boldsymbol{\sigma}$ is 1-connected.

The next theorems describe minimal norm multi-convex projections. But first a few comments are in order. By definition we always have $m \leq M$. Whenever $M=n$, we automatically assume $L \geq n$. Theorem 2.1 indicates that there are two possible situations (of interest to us) in which $m=M$, namely $m=M=n-1$ and $m=M=n$. These cases are actually " $k$ convex" shapes (regarded as specific multi-convex shapes); moreover these situations constitute somewhat extreme cases in the multi-convex realm. 
The case $m=M=n-1$ has been handled in [11]. For completeness, we give the result on minimality for this case in Theorem 2.2.

In the case $m=M=n$, the minimal shape-preserving projection problem is completely unsolved for $n \geq 2$ (the projection given in [8] partially solves the problem in the $n=2$ case). Indeed, it is conjectured in [11] that a minimal norm projection from $X=C^{L}[0,1]$ onto $V=\Pi_{n}$ preserves $n$-convexity for every $L=0,1, \ldots$ That is, in the case of $n$-convexity, the minimal shape-preserving projection problem is perhaps equivalent to the minimal projection problem. Therefore, this paper does not address this case.

There is one more exceptional case: $m=n-1$ and $M=n$. It turns out that the results concerning minimal shape-preserving projections in this case are similar to those for $m=M=n-1$ but the method of proof differs substantially from the approach in [11] as well as from that adopted here. Consequently, the $m=n-1, M=n$ case is handled in [28]. However, Theorem 2.2 below states the result for this case.

Throughout the remainder of this paper we will assume $n \geq 2$. In the $n=1$ case, there is a projection of norm one in $\mathcal{P}_{S \boldsymbol{\sigma}}\left(X, \Pi_{1}\right)$.

Theorem 2.2 (see [11] and [28]). Let $X=\left(C^{L}[0,1],\|\cdot\|_{L}\right)$. For fixed $n$ let $m=n-1 \leq M$. Then there exists $P_{m} \in \mathcal{P}_{S \boldsymbol{\sigma}}\left(X, \Pi_{n}\right)$ such that $\left\|P_{m}\right\|=3 / 2$ and $\left\|P_{m}\right\| \leq\|P\|$ for every $P \in \mathcal{P}_{S \boldsymbol{\sigma}}\left(X, \Pi_{n}\right)$.

Theorem 2.3. Let $X=\left(C^{L}[0,1],\|\cdot\|_{L}\right)$. For fixed $n$, assume $M \geq n-1$ and $\boldsymbol{\sigma}$ is 1-connected. Suppose $m=0$ and define $P_{0, n}=\sum_{i=0}^{n} u_{i} \otimes v_{i}$ where

$$
\begin{aligned}
& u_{i}=\delta_{0}^{i} \quad \text { for } i \neq n, \quad u_{n}=\delta_{1}^{n-1}, \\
& v_{i}=\frac{x^{i}}{i !} \quad \text { for } i \neq n-1, \quad v_{n-1}=\frac{x^{n-1}}{(n-1) !}-\frac{x^{n}}{n !} ;
\end{aligned}
$$

i.e.,

(2) $P_{0, n}=\delta_{0} \otimes 1+\delta_{0}^{1} \otimes \frac{x}{1 !}+\cdots+\delta_{0}^{n-1} \otimes\left(\frac{x^{n-1}}{(n-1) !}-\frac{x^{n}}{n !}\right)+\delta_{1}^{n-1} \otimes \frac{x^{n}}{n !}$.

Then $P_{0, n}$ has minimal norm in $\mathcal{P}_{S_{\boldsymbol{\sigma}}}\left(X, \Pi_{n}\right)$ and

$$
\left\|P_{0, n}\right\|=\sum_{k=0}^{n-1} \frac{1}{k !} .
$$

Moreover, in the case that $M=n-1$, we have $\left\{P_{0, n}\right\}=\mathcal{P}_{S_{\boldsymbol{\sigma}}}\left(X, \Pi_{n}\right)$.

Theorem 2.4. Let $X=\left(C^{L}[0,1],\|\cdot\|_{L}\right)$ and $X_{1}=\left(C^{L+1}[0,1],\|\cdot\|_{L+1}\right)$. For fixed integer $n$, assume $0<m<n-1 \leq M$ and $\boldsymbol{\sigma}$ is 1-connected. Let $Y \subset X$ denote the $(n+1)$-dimensional subspace spanned by $\left\{w_{0}, w_{1}, \ldots, w_{n}\right\}$, i.e., $Y=\left[w_{0}, w_{1}, \ldots, w_{n}\right]$. Let $P_{m, n}=\sum_{i=0}^{n} q_{i} \otimes w_{i}$ be a projection from $X$ onto $Y$ which preserves $S_{\boldsymbol{\sigma}}$, i.e., $P_{m, n} \in \mathcal{P}_{S_{\boldsymbol{\sigma}}}(X, Y)$. Define the operator 
$P_{m+1, n+1}$ on $X_{1}$ by

$$
\begin{aligned}
\left(P_{m+1, n+1} f\right)(x)= & \frac{f(0)+f(1)}{2}+\int_{0}^{x}\left(P_{m, n} f^{\prime}\right)(t) d t \\
& -\frac{1}{2} \int_{0}^{1}\left(P_{m, n} f^{\prime}\right)(t) d t
\end{aligned}
$$

where $f^{\prime}$ denotes the derivative of $f$. Then

$$
P_{m+1, n+1} \in \mathcal{P}_{S_{\widehat{\boldsymbol{\sigma}}}}\left(X_{1},\left[1, W_{0}, W_{1}, \ldots, W_{n}\right]\right)
$$

where

$$
W_{i}(t)=\int_{0}^{t} w_{i}(s) d s
$$

and $\widehat{\boldsymbol{\sigma}}$ is the 1-connected $(n+2)$-tuple such that $\max _{\widehat{\sigma}_{i}=1} i=M+1$ and $\min _{\widehat{\sigma}_{i}=1} i=m+1$. Moreover, if $\left\|P_{m, n}\right\| \geq 2$ then

$$
\left\|P_{m+1, n+1}\right\|=\left\|P_{m, n}\right\| \text {. }
$$

TheOREM 2.5. Let $k$ be a nonnegative integer. Let $X=\left(C^{L+k}[0,1],\|\cdot\|\right)$ where $\|\cdot\|$ is any norm such that

$$
\|\cdot\|_{2, L+k} \leq\|\cdot\| \leq\|\cdot\|_{L+k}
$$

where

$$
\begin{aligned}
\|f\|_{2, L+k} & =\max \left\{\max _{j=0, \ldots, L+k-1}\left\{\left|f^{(j)}(0)\right|,\left|f^{(j)}(1)\right|\right\},\left\|f^{(L+k)}\right\|_{\infty}\right\}, \\
\|f\|_{L+k} & =\max _{i=0, \ldots, L+k}\left\{\left\|f^{(i)}\right\|_{\infty}\right\} .
\end{aligned}
$$

Let $P_{k, n+k}$ denote the operator obtained by $k$ applications of (4) beginning with $P_{0, n}$ given in Theorem 2.3. Then $P_{k, n+k}$ is a minimal norm element of $\mathcal{P}_{S \boldsymbol{\sigma}}\left(X, \Pi_{n+k}\right)$ where $\boldsymbol{\sigma}$ is the 1-connected $(n+k+1)$-tuple such that $\max _{\sigma_{i}=1} i \geq n+k-1$ and $\min _{\sigma_{i}=1} i=k$.

In general, given two norms that are equivalent (but not proportional), we should not expect a projection that has minimal operator norm with respect to the first norm to be minimal in the operator norm determined by the second. From this viewpoint, Theorem 2.5 is quite surprising.

The proofs of Theorems 2.1, 2.3, 2.4 and 2.5 are contained in the sections that follow. We first verify the existence in Section 3. Then, in Section 4, we show how to calculate the norms of functionals from a particular family. This calculation will play a crucial role in Section 5, where we verify the shape-preserving properties and norm minimality of the constructed projections. 
3. Proof of existence. We employ results from [31] to establish existence. The relevant material from that paper is included below.

A cone $K$ in a Banach space is defined to be a convex set which is closed under nonnegative scalar multiplication. $K$ is said to be pointed if $K$ contains no lines through 0 .

For $\phi \in K$, let $[\phi]^{+}:=\{\alpha \phi \mid \alpha \geq 0\}$. We say $[\phi]^{+}$is an extreme ray of $K$ if $\phi=\phi_{1}+\phi_{2}$ with $\phi_{1}, \phi_{2} \in K$ implies $\phi_{1}, \phi_{2} \in[\phi]^{+}$. Let $E(K)$ denote the union of all extreme rays of $K$. When $K$ is a closed, pointed cone of finite dimension we always have $K=\operatorname{co}(E(K))$.

We say a finite (possibly) signed measure $\mu$ with support $E \subset X^{*}$ is a generalized representing measure for $\phi \in X^{*}$ if $\langle x, \phi\rangle=\int_{E}\langle s, x\rangle d \mu(s)$ for all $x \in X$. A nonnegative measure $\mu$ satisfying this equality is simply a representing measure.

Definition 3.1. Let $X$ be a Hausdorff topological vector space over $\mathbb{R}$ and let $X^{*}$ be the topological dual of $X$. We say that a pointed closed cone $K \subset X^{*}$ is simplicial if $K$ can be recovered from its extreme rays, (i.e., $K=\overline{\mathrm{co}}(E(K)))$ and the set of extreme rays of $K$ is independent (in the sense that any generalized representing measure for $x \in K$ supported on $E(K)$ must be a representing measure).

Proposition 3.1. A pointed closed cone $K \subset X^{*}$ of finite dimension d is simplicial iff $K$ has exactly d extreme rays.

For given $S_{\boldsymbol{\sigma}}$, we define its dual cone as

$$
S^{*}=\left\{u \in X^{*} \mid u(f) \geq 0 \forall f \in S_{\boldsymbol{\sigma}}\right\} .
$$

Note that for each $S_{\boldsymbol{\sigma}}$, the cone dual $S^{*}$ is simplicial with $[\phi]^{+} \in E\left(S^{*}\right)$ iff $\phi=\varrho \delta_{t}^{k}$ where $\varrho>0, t \in[0,1]$ and $k \in\{0,1, \ldots, L\}$. The result we will need is the following.

Theorem 3.1. Let $X=\left(C^{L}[0,1],\|\cdot\|_{L}\right)$. Then $\mathcal{P}_{S_{\boldsymbol{\sigma}}}\left(X, \Pi_{n}\right) \neq \emptyset$ if and only if the cone $S_{\left.\right|_{\Pi_{n}} ^{*}}^{*}$ is simplicial.

Proof of Theorem 2.1. Throughout this proof we denote the dual cone of $S_{\sigma}$ by $S^{*}$, and $\Pi_{n}$ by $V$.

$(\Leftarrow)$ We verify that $S_{\left.\right|_{V}}^{*}$ is simplicial. Note that for each $t \in[0,1]$ and each integer $j \in[m, M]$ the functional $\delta_{t}^{j}$ belongs to an extreme ray of $S^{*}$. Therefore, we need only demonstrate that a simplicial subcone of $S_{\left.\right|_{V}}^{*}$ contains all restrictions $\left(\delta_{t}^{j}\right)_{\left.\right|_{V}}$; this will then imply that $S_{\left.\right|_{V}}^{*}$ is itself simplicial. To this end, we fix for $V$ the basis $\left\{v_{i}\right\}_{i=0}^{n}$ where $v_{i}=x^{i} / i$ ! and embed $S_{\left.\right|_{V}}^{*}$ into (the positive orthant of) $\mathbb{R}^{n+1}$ via the identification 


$$
\phi_{\left.\right|_{V}} \equiv\langle\boldsymbol{v}, \phi\rangle=\left(\begin{array}{c}
\left\langle v_{0}, \phi\right\rangle \\
\left\langle v_{1}, \phi\right\rangle \\
\vdots \\
\left\langle v_{n}, \phi\right\rangle
\end{array}\right)
$$

with this understanding we will regard $S_{\left.\right|_{V}}^{*} \subset \mathbb{R}^{n+1}$. Let us now consider the case $M=n-1$ (we will see that the $M=n$ case follows in the same way). Notice that, for an integer $j \in[m, n-1]$ and $t \in[0,1]$, we have

$$
\left(\delta_{t}^{j}\right)_{\left.\right|_{V}}=\left(\begin{array}{c}
0_{1} \\
\vdots \\
0_{j} \\
1 \\
t / 1 ! \\
\vdots \\
t^{k-j} /(k-j) ! \\
\vdots \\
t^{n-j} /(n-j) !
\end{array}\right)
$$

Denote by $\boldsymbol{e}_{i}$ the vector $\left(0_{1}, \ldots, 0_{i-1}, 1,0_{i+1}, \ldots, 0_{n+1}\right)^{T} \in \mathbb{R}^{n+1}$; from (6) it is clear that, for every integer $j \in[m, n-1], \boldsymbol{e}_{j+1} \in S_{\left.\right|_{V}}^{*}$ (given by $\left(\delta_{0}^{j}\right)_{\left.\right|_{V}}$ ) as well as $\boldsymbol{e}_{n}+\boldsymbol{e}_{n+1} \in S_{\left.\right|_{V}}^{*}$ (given by $\left.\left(\delta_{1}^{n-1}\right)_{\left.\right|_{V}}\right)$. Moreover, for every integer $j \in[m, n-1]$ we have

$$
\begin{aligned}
\left(\delta_{t}^{j}\right)_{\left.\right|_{V}}= & \sum_{k=j}^{n-2} \frac{t^{k-j}}{(k-j) !} \boldsymbol{e}_{k+1}+\frac{t^{n-j-1}}{(n-j-1) !}\left(1-\frac{t}{n-j}\right) \boldsymbol{e}_{n} \\
& +\frac{t^{n-j}}{(n-j) !}\left(\boldsymbol{e}_{n}+\boldsymbol{e}_{n+1}\right) .
\end{aligned}
$$

Since the coefficient functions of $\boldsymbol{e}_{n}+\boldsymbol{e}_{n+1}$ and each $\boldsymbol{e}_{k}, k=j, \ldots, n$, are nonnegative, we see that $S_{\left.\right|_{V}}^{*}$ is simplicial. In the case that $M=n$, we again note from (6) that $\boldsymbol{e}_{j} \in S_{\left.\right|_{V}}^{*}$ for every integer $j=m+1, \ldots, n+1$ and thus $S_{\left.\right|_{V}}^{*}$ is also simplicial.

$(\Rightarrow)$ Assume $\mathcal{P}_{S_{\boldsymbol{\sigma}}} \neq \emptyset$. By Theorem 3.1, we know $S_{\left.\right|_{V}}^{*}$ is simplicial. Let $E=\left\{\left[x_{1}\right]^{+}, \ldots,\left[x_{d}\right]^{+}\right\}$be the set of extreme rays of $S_{\left.\right|_{V}}^{*}$. We first show that $M$ must be at least $n-1$. Suppose, to the contrary, that $M \leq n-2$. For convenience, fix for $V$ the basis $\left\{v_{i}\right\}_{i=0}^{n}$ where 


$$
v_{i}= \begin{cases}x^{i} / i !, & i<M, \\ (i-M) ! / i ! x^{i}, & i \geq M .\end{cases}
$$

For $t \in[0,1]$, let $\Delta_{t}:=\left(\delta_{t}^{M}\right)_{\left.\right|_{V}}$ and, via the embedding into $\mathbb{R}^{n+1}$ described above, notice that $\Delta_{t}=\left(0_{1}, \ldots, 0_{M}, 1, t, t^{2}, \ldots, t^{n-M}\right)$. Consider the subcone $K$ of $S_{\left.\right|_{V}}^{*} \subset \mathbb{R}^{n+1}$ generated by the rays $\left[\Delta_{t}\right]^{+}$:

$$
K=\overline{\mathrm{co}}\left(\left\{\left[\Delta_{t}\right]^{+} \mid t \in[0,1]\right\}\right) .
$$

Then $K$ has infinitely many extreme rays since each $\Delta_{t}$ is an extreme point of $C=\overline{\mathrm{co}}\left(\left\{\Delta_{t} \mid t \in[0,1]\right\}\right)$ ( $C$ is a translate of the convex hull of the moment curve $\left.\left(t, t^{2}, \ldots, t^{n-M}\right)\right)$. If $m=M$ then we have an immediate contradiction; assume then that $0 \leq m<M$. Consequently, $E$ cannot lie entirely in $K$; without loss assume $\left\{\left[x_{1}\right]^{+}, \ldots,\left[x_{k}\right]^{+}\right\}=E-(E \cap K)$. Since each such ray is extreme, for each $i=1, \ldots, k$ we must have $\left[x_{i}\right]^{+}=\left[\left(\delta_{t}^{j}\right)_{\left.\right|_{V}}\right]^{+}$for some $t \in[0,1]$ and some integer $j \in[m, M-1]$. But because $j<M$, we see that every (nonzero) element $\left(a_{1}, \ldots, a_{n+1}\right)^{T}$ from $\operatorname{co}\left(\left\{\left[x_{1}\right]^{+}, \ldots,\left[x_{k}\right]^{+}\right\}\right)$has at least one nonzero entry in the first $M$ coordinates, i.e., there exists an integer $s \in[0, M]$ such that $a_{s} \neq 0$. This implies $\operatorname{co}\left(\left\{\left[x_{1}\right]^{+}, \ldots,\left[x_{k}\right]^{+}\right\}\right) \cap K=\emptyset$. Thus $K \subset S_{\left.\right|_{V}}^{*}$ cannot be contained in a simplicial subcone of $S_{\left.\right|_{V}}^{*}$, and this contradicts the fact that $S_{\left.\right|_{V}}^{*}$ simplicial. Therefore $M \geq n-1$.

We now show $\boldsymbol{\sigma}$ is 1-connected. Suppose it is not; let $Z:=\max \{i \mid$ $\sigma_{i+1}=0$ and $\left.i<M\right\}$ ( $Z$ marks the location of the last 1 in $\boldsymbol{\sigma}$ before the last break of the sequence of 1's). For convenience fix for $V$ the basis in (7) using $Z$ rather than $M$. Similar to the above, define $\Delta_{t}:=\left(\delta_{t}^{Z}\right)_{\left.\right|_{V}}$ and $K$ as in (8). Then $K$ has infinitely many extreme rays and thus, as before, the set of extreme rays of $S_{\left.\right|_{V}}^{*}, E=\left\{\left[x_{1}\right]^{+}, \ldots,\left[x_{d}\right]^{+}\right\}$, cannot lie entirely in $K$. Every $x_{i}$ must be of the form $\left(\delta_{t}^{j}\right)_{\left.\right|_{V}}$ for some $t \in[0,1]$ and some integer $j$ as prescribed by $\boldsymbol{\sigma}$. The convex hull of $\left\{\left[x_{i}\right]^{+} \mid x_{i}=\left(\delta_{t}^{j}\right)_{\left.\right|_{V}}\right.$ for $j \geq Z+2\}$ misses every (nonzero) element of $K$ since every element $\left(a_{1}, \ldots, a_{n+1}\right)$ of this convex hull has $a_{Z+1}=0$. Similarly, the convex hull of $\left\{\left[x_{i}\right]^{+} \mid x_{i}=\left(\delta_{t}^{j}\right)_{\left.\right|_{V}}\right.$ for $\left.j \leq Z-1\right\}$ misses every (nonzero) element of $K$ since every element $\left(a_{1}, \ldots, a_{n+1}\right)$ of this convex hull has $a_{s} \neq 0$ for some integer $s \in[m, Z-1]$. If $\boldsymbol{\sigma}$ is not 1-connected then we have exhausted all possible choices for $x_{i}$ (in particular, there is not $x_{i}$ of the form $\left(\delta_{t}^{Z+1}\right)_{\left.\right|_{V}}$ ) and we find that $S_{\left.\right|_{V}}^{*}$ cannot be simplicial. This contradiction shows that $\boldsymbol{\sigma}$ is 1 -connected.

4. Results on the geometry of $B\left(C^{L}[0,1],\|\cdot\|_{L}\right)$. We start with two well known lemmas, whose straightforward proofs are omitted.

Lemma 4.1. Let $(X,\|\cdot\|)$ be a normed space. Suppose that $\left(\|\cdot\|_{k}\right)$ is a sequence of equivalent norms on $X$ such that 


$$
\|x\|_{k}\left(1-a_{k}\right) \leq\|x\| \leq\left(1+a_{k}\right)\|x\|_{k}
$$

for any $x \in X$. Assume $a_{k} \rightarrow 0$. Let $\mathcal{L}_{k}(X)$ denote the space of linear operators defined on $X$, continuous with respect to $\|\cdot\|$, with the operator norm induced by $\|\cdot\|_{k}$. Then for any $T \in \mathcal{L}(X)$,

$$
\|T\|_{k} \rightarrow\|T\|,
$$

where $\|T\|$ denotes the operator norm of $T$ induced by $\|\cdot\|$.

Lemma 4.2. Let $(X,\|\cdot\|)$ be a normed space and let $\|\cdot\|_{k}$ be a sequence of norms on $X$ satisfying $(9)$ such that $a_{k} \rightarrow 0$. Let $X_{k}^{*}$ denote the dual space $X^{*}$ equipped with the norm induced by $\|\cdot\|_{k}$. Then for any $f \in X^{*}$,

$$
\|f\|_{k} \rightarrow\|f\|
$$

where $\|f\|$ denotes the norm of $f$ in $X^{*}$.

Definition 4.1. Let $\left\{t_{i}\right\}$ be a countable, dense subset of $[0,1]$ such that $t_{0}=0$ and $t_{1}=1$. For $k \in \mathbb{N}$ define a norm $\|\cdot\|_{k, L}$ on $C^{L}[0,1]$ by

$$
\|f\|_{k, L}=\max _{i=0, \ldots, L} A_{i k}(f)
$$

where

$$
\begin{aligned}
& A_{i k}(f)=\max _{j=1, \ldots, k}\left|f^{(i)}\left(t_{j}\right)\right| \quad \text { for } i=0, \ldots, L-1, \\
& A_{L k}(f)=\left\|f^{(L)}\right\|_{\infty} .
\end{aligned}
$$

Lemma 4.3. Let $\|\cdot\|_{k, L}$ be as in Definition 4.1. Then for any $\varepsilon>0$ there exists $k_{0} \in \mathbb{N}$ such that for any $f \in C^{L}[0,1]$ and $k \geq k_{0}$,

$$
\|f\|_{k, L} \leq\|f\|_{L} \leq(1+\varepsilon)\|f\|_{k, L}
$$

where

$$
\|f\|_{L}=\max _{i=0, \ldots, L}\left\{\left\|f^{(i)}\right\|_{\infty}\right\} .
$$

Proof. Fix $k \in \mathbb{N}, k \geq 3$. Without loss of generality, we can assume that

$$
1=t_{0}<t_{2}<\cdots<t_{k}<t_{1}=1 .
$$

Set

$$
\Delta_{k}=\max \left\{t_{2}-t_{0}, t_{1}-t_{k}, t_{j}-t_{j-1} \mid j=3, \ldots, k\right\} .
$$

By the density of $\left\{t_{j}\right\}, \Delta_{k} \rightarrow 0$. Fix $k_{0} \in \mathbb{N}$ such that

$$
\left(1+\Delta_{k}\right)^{L+1}<1+\varepsilon
$$

for $k \geq k_{0}$. Take any $k \geq k_{0}$. Fix $f \in X$. First we show that

$$
\left\|f^{(L-1)}\right\|_{\infty} \leq\left(1+\Delta_{k}\right)\|f\|_{k, L} .
$$

Let $t \in[0,1]$ be so chosen that $\left\|f^{(L-1)}\right\|_{\infty}=\left|f^{(L-1)}(t)\right|$. Then $t \in\left[t_{0}, t_{2}\right]$ or $t \in\left[t_{k}, t_{1}\right]$ or $t \in\left[t_{i}, t_{i+1}\right]$ for some $i=2, \ldots, k$. Hence by the definition of $\Delta_{k}$ and the mean value theorem, for properly chosen $t_{i}, i=0, \ldots, k$,

$$
\left|f^{(L-1)}(t)-f^{(L-1)}\left(t_{i}\right)\right| \leq\left\|f^{(L)}\right\|_{\infty}\left|t-t_{i}\right|
$$




\section{Hence}

$$
\left\|f^{(L-1)}\right\|_{\infty}=\left|f^{(L-1)}(t)\right| \leq\left|f^{(L-1)}\left(t_{i}\right)\right|+\left\|f^{(L)}\right\|_{\infty} \Delta_{k} \leq\|f\|_{k, L}\left(1+\Delta_{k}\right),
$$

which proves (11). Analogously, by the mean value theorem,

$$
\left\|f^{(L-j)}\right\|_{\infty} \leq\left(1+\Delta_{k}\right)^{j}\|f\|_{k, L}
$$

for $j=0, \ldots, L$. Hence

$$
\max _{i=0, \ldots, L}\left\{\left\|f^{(i)}\right\|_{\infty}\right\} \leq\left(1+\Delta_{k}\right)^{L+1}\|f\|_{k, L},
$$

which gives $\|f\|_{L} \leq(1+\varepsilon)\|f\|_{k, L}$ for $k \geq k_{0}$, as required. Then

Theorem 4.1. Let $X=\left(C^{L}[0,1],\|\cdot\|_{L}\right)$ and $X_{k}=\left(C^{L}[0,1],\|\cdot\|_{k, L}\right)$.

$$
\begin{aligned}
& \operatorname{ext}\left(B\left(X^{*}\right)\right) \subset\left\{ \pm \delta_{t}^{i} \mid i=0, \ldots, L, t \in[0,1]\right\}, \\
& \operatorname{ext}\left(B\left(X_{k}^{*}\right)\right) \subset\left\{ \pm \delta_{t_{j}}^{i} \mid i=0, \ldots, L-1, j=0, \ldots, k\right\} \cup\left\{\delta_{t}^{L} \mid t \in[0,1]\right\} .
\end{aligned}
$$

Proof. Note that $X$ can be isometrically embedded in $Z=(C[0,1])^{L+1}$ with the norm

$$
\left\|\left(f_{1}, \ldots, f_{L+1}\right)\right\|=\max _{i=1, \ldots, L+1}\left\|f_{i}\right\|_{\infty} .
$$

The embedding is given by the formula

$$
T(f)=\left(f, f^{(1)}, \ldots, f^{(L)}\right) .
$$

Note that

$$
\operatorname{ext}\left(B\left(Z^{*}\right)\right)=\left\{\left(0, \ldots, \pm \delta_{t}, 0, \ldots, 0\right): t \in[0,1]\right\} .
$$

To show our claim we prove that $X$ is a weakly separating subspace of $Z$. Recall that a linear subspace $V$ of a Banach space $W$ is called weakly separating if any point from $\operatorname{ext}\left(B\left(V^{*}\right)\right)$ has only one Hahn-Banach extension in $B\left(W^{*}\right)$.

So assume $x^{*} \in \operatorname{ext}\left(B\left(X^{*}\right)\right)$. Set

$$
K=\left\{f \in B\left(Z^{*}\right): f_{\left.\right|_{X}}=x^{*}\right\} .
$$

We show that $K$ consists of exactly one element from $\operatorname{ext}\left(B\left(Z^{*}\right)\right)$. It is easy to see that $K$ is a convex, weak* closed subset of $B\left(Z^{*}\right)$. By the Banach-Alaoglu theorem $K$ is weak* compact. By the Krein-Milman theorem $\operatorname{ext}(K) \neq \emptyset$. First we show that $\operatorname{ext}(K) \subset \operatorname{ext}\left(B\left(Z^{*}\right)\right)$. Take any $g \in \operatorname{ext}(K)$ and assume $g=\left(g_{1}+g_{2}\right) / 2$, where $g_{1}, g_{2} \in B\left(Z^{*}\right)$. Then

$$
f=g_{X}=\frac{\left(g_{1}\right)_{\left.\right|_{X}}+\left(g_{2}\right)_{\left.\right|_{X}}}{2} .
$$

Since $f \in \operatorname{ext}\left(B\left(X^{*}\right)\right)$, we have $\left(g_{1}\right)_{\left.\right|_{X}}=f$ and $\left(g_{2}\right)_{\left.\right|_{X}}=f$. Hence $g_{1}, g_{2} \in K$. Since $g \in \operatorname{ext}(K)$, it follows that $g_{1}=g_{2}$, as required. 
Now assume on the contrary that $K$ consists of more than one element. Then we can find at least two different points of $\operatorname{ext}\left(B\left(Z^{*}\right)\right)$,

$$
z_{1}= \pm\left(0, \ldots,\left(\delta_{s}\right)_{i}, 0, \ldots, 0\right), \quad z_{2}= \pm\left(0, \ldots,\left(\delta_{t}\right)_{j}, 0, \ldots, 0\right),
$$

belonging to $\operatorname{ext}(K)$. Hence $\left(z_{1}\right)_{\left.\right|_{X}}=\left(z_{2}\right)_{\left.\right|_{X}}$. But if $i<j$ then taking $f_{i}(t)=$ $t^{i}$ we get (via isometric embedding) $z_{1}\left(f_{i}\right)=1$ and $z_{2}\left(f_{i}\right)=0$. Hence $i=j$. If $s \neq t$ then taking $f_{i}(t)=t^{i+1}$ we also get $z_{1}\left(f_{i}\right) \neq z_{2}\left(f_{i}\right)$. Finally, if

$$
z_{1}=\left(0, \ldots,\left(\delta_{t}\right)_{i}, 0, \ldots, 0\right), \quad z_{2}=-\left(0, \ldots,\left(\delta_{t}\right)_{i}, 0, \ldots, 0\right),
$$

then also $z_{1}\left(f_{i}\right) \neq z_{2}\left(f_{i}\right)$, where $f_{i}(t)=t^{i}$. Consequently, $z_{1}=z_{2}$; a contradiction. Hence $K$ consists of exactly one element $z$. By the previous reasoning $z \in \operatorname{ext}\left(B\left(Z^{*}\right)\right.$ ). Consequently, (via isometric embedding) $x^{*}= \pm \delta_{t}^{i}$ for some $t \in[0,1]$ and $i=0, \ldots, L$, as required.

Now we consider the case of $X_{k}$. Note that $X_{k}$ can be isometrically embedded into

$$
C=\mathbb{R}^{(k+1) L} \times C[0,1]
$$

equipped with the norm

$$
\left\|\left(r_{1}, \ldots, r_{(k+1) L}, f\right)\right\|=\max \left\{\left|r_{i}\right|(i=1, \ldots,(k+1) L),\|f\|_{\infty}\right\} .
$$

The embedding is given by

$$
T(f)=\left(f\left(t_{j}\right)^{(i)}(i=0, \ldots, L-1, j=0, \ldots, k), f^{(L)}\right) .
$$

Reasoning in the same way as in the case of $X$ we get our result.

Theorem 4.2. Let $X_{k}=\left(C^{L}[0,1],\|\cdot\|_{k, L}\right), L \geq 2$. Set

$$
g=\frac{\sum_{i=0}^{L-1} \delta_{0}^{i}}{L} \in\left(X_{k}\right)^{*} \text {. }
$$

Then $\|g\|=1$.

Proof. Without loss of generality, we can assume that

$$
0=t_{0}<t_{1}<\cdots<t_{k}=1 .
$$

It is clear that $\|g\| \leq 1$. Assume that $\|g\|<1$. Then $\|b g\|=1$ for some $b>1$, since $g \neq 0$. By Theorem 4.1 and the Krein-Milman theorem,

$$
b g=\sum_{i=0}^{L-1} \sum_{j=0}^{k} a_{i j} \delta_{t_{j}}^{i}+u .
$$

Here $u$ is a Radon measure on $[0,1]$ acting as a functional on $X_{k}$ as

$$
\widehat{u}(f)=\int_{[0,1]} f^{(L)}(t) d u(t)
$$


and

$$
\sum_{i=0}^{L-1} \sum_{j=0}^{k}\left|a_{i j}\right|+\|u\|=1
$$

where $\|u\|$ denotes the total variation of $u$.

First we show that $u=0$. Assume that $u \neq 0$. Then

$$
u=b g-\sum_{i=0}^{L-1} \sum_{j=0}^{k} a_{i j} \delta_{t_{j}}^{i}
$$

Note that $u=\sum_{l=0}^{k-1} u_{l}$, where $u_{l}$ is the Radon measure defined by $u_{l}(A)=$ $u\left(A \cap E_{l}\right)$ for $l=0, \ldots, k-1$, where $E_{l}=\left[t_{l}, t_{l+1}\right)$ for $l=0, \ldots, k-2$ and $E_{k-1}=\left[t_{k-1}, 1\right]$.

Let us first assume that for every $l=0, \ldots, k-1, u_{l}=c_{l} m_{l}$, where $m_{l}$ is the Lebesgue measure on $E_{l}$ and $c_{l} \in \mathbb{R}$. By the fundamental theorem of calculus,

$$
m_{l}(f)=c_{l} \int_{E_{l}} f^{(L)}(s) d s=c_{l}\left(f^{(L-1)}\left(t_{l+1}\right)-f^{(L-1)}\left(t_{l}\right)\right)
$$

for $l=0, \ldots, k-1$. By the Hermite interpolation theorem there exists a polynomial $p$ such that

$$
\begin{array}{rlrl}
p^{(i)}(0) & =0 & & \text { for } i=0, \ldots, L-1, \\
p^{(i)}\left(t_{j}\right) & =\operatorname{sgn}\left(a_{i j}\right) & & \text { for } i=0, \ldots, L-2, j=1, \ldots, k, \\
p^{(L-1)}\left(t_{j}\right) & =\operatorname{sgn}\left(a_{L-1, j}-c_{j-1}+c_{j}\right) \quad \text { for } j=1, \ldots, k .
\end{array}
$$

Observe that

$$
0=(b g)(p)=\sum_{i=0}^{L-2} \sum_{j=1}^{k}\left|a_{i j}\right|+\sum_{j=1}^{k}\left|a_{L-1, j}+c_{j-1}-c_{j}\right| .
$$

Hence all coefficients in the above sum are 0 . But this implies that

$$
b g=\sum_{i=0}^{L-2} a_{i, 0} \delta_{0}^{i}+\left(a_{L-1,0}-c_{0}\right) \delta_{0}^{L-1} .
$$

Since $b>1$ and the set $\left\{\delta_{0}^{i}: i=0, \ldots, L-1\right\}$ is linearly independent, this leads to a contradiction with (13).

So assume that there exists $l \in\{0, \ldots, k-1\}$ such that $u_{l} \neq 0$ and $u_{l}$ is not a constant multiple of $m_{l}$. By (12),

$$
u(f)=\int_{[0,1]} f^{(L)}(t) d u(t)=0
$$

for any $f \in X_{k}$ satisfying

$$
f^{(i)}\left(t_{j}\right)=0 \quad \text { for } i=0, \ldots, L-1, j=0, \ldots, k .
$$


Fix any $f \in X_{k}$ satisfying (14). Suppose there exist $D_{1} \subset E_{l}$ and $D_{2} \subset E_{l}$ such that

$$
u\left(D_{1}\right) \cdot u\left(D_{2}\right)<0 .
$$

Assume $u\left(D_{1}\right)>0$. Modifying $D_{1}$ and $D_{2}$ if necessary, we can assume that $D_{1} \cap D_{2}=\emptyset$. By the properties of Radon measures there exist two disjoint subintervals (denote them also by $D_{1}, D_{2}$ ) of $E_{l}$ of the same length $c>0$ satisfying (16). Set

$$
h_{L}=\chi_{D_{1}}-\chi_{D_{2}} \text {. }
$$

We now modify $h_{L}$ to a continuous function $h_{L}^{l}$ on $[0,1]$ with support contained in $D_{1} \cup D_{2}$. To do this fix $l \in \mathbb{N}$ such that $c-2 / l>0$. Assume that $D_{1}=\left(s_{1}, s_{2}\right), D_{2}=\left(w_{1}, w_{2}\right)$ and $s_{2}<w_{1}$. Set $h_{L}^{l}(t)=h_{L}(t)$ if $t \notin D_{1} \cup D_{2}$, $h_{L}^{l}(t)=1$ if $t \in\left(s_{1}+1 / l, s_{2}-1 / l\right), h_{L}^{l}(t)=-1$ if $t \in\left(w_{1}+1 / l, w_{2}-1 / l\right)$ and extend it linearly for other $t$. Note that, for Lebesgue measure $m$,

$$
\int_{[0,1]} h_{L}^{l}(t) d m(t)=0
$$

and $l$ can be taken so large that

$$
\int_{[0,1]} h_{L}^{l}(t) d u(t)>0
$$

Set $H_{L}(t)=h_{L}^{l}(t)$, where $l$ is so chosen that (16) and (19) are satisfied. Set

$$
H_{j-1}(t)=\int_{[0, t]} H_{j}(s) d m(s) \quad \text { for } j=L, L-1, \ldots, 1 .
$$

Define

$$
G=f+H_{0}
$$

By the construction of $H_{L}$,

$$
\int_{E_{l}} H_{j}(s) d m(s)=0 \quad \text { for } j=1, \ldots, L .
$$

Consequently,

$$
G^{(i)}\left(t_{j}\right)=0 \quad \text { for } i=0, \ldots, L-1, j=0, \ldots, k
$$

and thus $G$ satisfies (15). Hence it should satisfy (14)-but this contradicts (19).

To end the proof that $u=0$, assume that $u_{l}$ does not satisfy (16). Hence $u_{l}$ or $-u_{l}$ is a nonzero measure on $E_{l}$ which is not a constant multiple of the Lebesgue measure of $E_{l}$. Without loss of generality we can assume that $u_{l}$ is a measure. By the above condition $u_{l}$ is not a Haar measure of $E_{l}$. Hence there exist an open interval $D_{1} \subset E_{l}$ and $t>0$ such that

$$
u_{l}\left(D_{1}\right) \neq u_{l}\left(D_{1}+t\right) \text {. }
$$


It follows that there exist two open disjoint intervals $D_{1}, D_{2} \subset E_{l}$ of the same length $c>0$ satisfying

$$
u_{l}\left(D_{1}\right) \neq u_{l}\left(D_{2}\right)
$$

Since obviously $m\left(D_{1}\right)=m\left(D_{2}\right)=c$, reasoning as above and replacing (16) by (20) we get a contradiction with (19). This finally shows that $u=0$.

Hence (12) reduces to

$$
b g=\sum_{i=0}^{L-1} \sum_{j=0}^{k} a_{i j} \delta_{t_{j}}^{i} .
$$

By the Hermite interpolation theorem there exists a polynomial $p$ such that

$$
\begin{array}{ll}
p^{(i)}(0)=0 & \text { for } i=0, \ldots, L-1, \\
p^{(i)}\left(t_{j}\right)=\operatorname{sgn}\left(a_{i j}\right) & \text { for } i=0, \ldots, L-1, j=1, \ldots, k .
\end{array}
$$

Observe that

$$
0=(b g)(p)=\sum_{i=0}^{L-1} \sum_{j=1}^{k}\left|a_{i j}\right| .
$$

Hence $a_{i j}=0$ for $j=1, \ldots, k$ and $i=0, \ldots, L-1$. By $(21)$,

$$
\sum_{i=0}^{L-1}\left(b / n-a_{i, 0}\right) \delta_{0}^{i}=0 .
$$

By the linear independence of the functionals $\delta_{0}^{i}, i=0, \ldots, L-1$, we get $a_{i, 0}=b / n$ for $i=0, \ldots, L-1$. Hence

$$
\sum_{i=0}^{L-1} a_{i, 0}=b>1,
$$

contrary to (13). The proof is complete.

Corollary 4.1. Let $X=\left(C^{L}[0,1],\|\cdot\|_{L}\right)$. Set

$$
g=\frac{\sum_{i=0}^{L} \delta_{0}^{i}}{L+1} \in X^{*}
$$

Then $\|g\|=1$.

Proof. Applying Theorem 4.2 to $Y_{k}=\left(C^{L+1}[0,1],\|\cdot\|_{k, L+1}\right)$ and $g$ we get $\|g\|_{Y_{k}^{*}}=1$. By Lemma 4.2, Lemma 4.3 and Theorem 4.2,

$$
\|g\|_{Y^{*}}=1
$$

where $Y=\left(C^{L+1}[0,1],\|\cdot\|_{L+1}\right)$. Hence, by the weak ${ }^{*}$ density of $Y$ in $Y^{* *}$, there exists a sequence $\left\{f_{k}\right\} \subset Y$ with

$$
\left\|f_{k}\right\|_{L+1}=\max _{i=0, \ldots, L+1}\left\|f_{k}^{(i)}\right\|_{\infty}=1
$$


such that $g\left(f_{k}\right) \rightarrow 1$. Note that $f_{k} \in X$ and

$$
\left\|f_{k}\right\|_{L}=\max _{i=0, \ldots, L}\left\|f^{(i)}\right\|_{\infty} \leq\left\|f_{k}\right\|_{L+1}=1 .
$$

Hence $\|g\|=1$, as required.

Lemma 4.4. Let $X=\left(C^{L}[0,1],\|\cdot\|_{L}\right)$. Fix an integer $k \in[0, L]$ and set

$$
g=\sum_{i=0}^{k} \delta_{0}^{i}+\delta_{1}^{k} \in X^{*} .
$$

Set

$$
W=\left\{F \in X^{* *} \mid F(g)=k+2 \text { and }\|F\|=1\right\} .
$$

Then $W \neq \emptyset$.

Proof. First consider the case $k=L$. By Corollary 4.1, there exists $F \in\left(X^{L}\right)^{* *}$ with $\|F\|=1$ such that $F\left(\delta_{0}^{i}\right)=1$ for $i=0, \ldots, k$. By the weak* $^{*}$ density of $X$ in $X^{* *}$, there exists a sequence $\left\{f_{j}\right\} \subset X$ with $\left\|f_{j}\right\|_{L} \leq 1$ such that $f_{j}^{(i)}(0) \rightarrow 1$ for $i=0, \ldots, k$. Define a sequence $\left\{g_{j}^{(k)}\right\}$ of continuous functions by $g_{j}^{(k)}(t)=f_{j}^{(k)}(t)$ if $t \in[0,1-1 / j], g_{j}^{(k)}(1)=1$, extended linearly onto the interval $[1-1 / j, 1]$. Note that for any $j \in \mathbb{N}$,

$$
g_{j}^{(k)}=f_{j}^{(k)}+h_{j}^{(k)}
$$

where $h_{j}^{(k)}(t)=0$ for $t \in[0,1-1 / j]$ and $\left\|h_{j}^{(k)}\right\|_{\infty} \leq 2$. Let

$$
h_{j}^{(i-1)}(t)=\int_{0}^{t} h_{j}^{(i)}(s) d s
$$

for $i=k, k-1, \ldots, 1$. Set

$$
g_{j}=f_{j}+h_{j}^{(0)} .
$$

By the mean value theorem,

$$
\left\|g_{j}^{(i)}\right\|_{\infty} \leq 1+2 / j \quad \text { for } i=0, \ldots, k-1
$$

and

$$
\left\|g_{j}^{(k)}\right\|_{\infty} \leq 1
$$

by definition. Hence $\left\|g_{j}\right\| \leq 1+2 / j$ for $j \in \mathbb{N}$. By the Banach-Alaoglu theorem $\left\{g_{j}\right\}$ has a cluster point $F_{1} \in X^{* *}$ with $\left\|F_{1}\right\|=1$. It is clear by definition of $g_{j}$ that $F_{1} \in W$. If $k<L$, by the Ascoli-Arzelà theorem applied to $\left\{f_{j}^{(k)}\right\}$ there exists a subsequence $\left\{j_{l}\right\}$ such that $f_{j_{l}}^{(k)}(1) \rightarrow 1$. Then we can proceed as in the previous case. Set

Theorem 4.3. Let $X=\left(C^{L}[0,1],\|\cdot\|_{L}\right)$ and fix an integer $k \in[0, L-1]$.

$$
W=\left\{F \in X^{* *} \mid F\left(\delta_{0}^{k}\right)=1,\|F\|=1\right\} .
$$


Assume $u$ is a Borel measure on $[0,1]$. Define $u^{k} \in X^{*}$ by

$$
u^{k}(f)=\int_{[0,1]} f^{(k)}(t) d u(t)
$$

Then for any $F \in W$,

$$
F\left(u^{k}\right) \geq 0 \text {. }
$$

Proof. Fix $F \in W$ and a Borel measure $u$. By the Goldstine theorem there exists a sequence $\left\{f_{l}\right\} \subset X$ with $\left\|f_{l}\right\|_{L} \leq 1$ such that

$$
f_{l}\left(\delta_{0}^{k}\right)=f_{l}^{(k)}(0) \rightarrow F\left(\delta_{0}^{k}\right)=1
$$

and

$$
f_{l}\left(u^{(k)}\right)=\int_{[0,1]} f_{l}^{(k)}(t) d u(t) \rightarrow F\left(u^{(k)}\right) .
$$

In particular, $\left\|f_{l}^{(k+1)}\right\|_{\infty} \leq 1$. Hence by the mean value theorem for any $s, t \in[0,1]$ and $l \in \mathbb{N}$,

$$
\left|f_{l}^{(k)}(s)-f_{l}^{(k)}(t)\right| \leq|t-s| .
$$

Also $\left\|f_{j}^{(k)}\right\|_{\infty} \leq 1$. By the Ascoli-Arzelà theorem, passing to a subsequence if necessary, we can assume that there exists $f \in C[0,1]$ such that

$$
\left\|f_{l}^{(k)}-f\right\|_{\infty} \rightarrow 0
$$

Now we show that $f(t) \geq 0$ for any $t \in[0,1]$. By $(23), f(0)=1$. Assume, on the contrary, that there exists $t_{0} \in(0,1]$ such that $f\left(t_{0}\right)<0$. By $(23)$ there exists $\delta>0$ such that

$$
\left|f_{l}^{(k)}(0)-f_{l}^{(k)}\left(t_{0}\right)\right|>1+\delta
$$

for $l \geq l_{0}$. By the mean value theorem,

$$
1+\delta<\left|f_{l}^{(k)}(0)-f_{l}^{(k)}\left(t_{0}\right)\right| \leq\left\|f_{l}^{(k+1)}\right\|_{\infty} t_{0} \leq 1 ;
$$

a contradiction.

Hence $f(t) \geq 0$ for any $t \geq 0$. By (25),

$$
f_{l}\left(u^{(k)}\right)=\int_{[0,1]} f_{l}^{(k)}(t) d u(t) \rightarrow \int_{[0,1]} f(t) d u(t) \geq 0,
$$

since $f$ is nonnegative and $u$ is a measure. By (24),

$$
F\left(u^{(k)}\right)=\int_{[0,1]} f(t) d u(t) \geq 0 .
$$

Theorem 4.4. Let $X=\left(C^{L}[0,1],\|\cdot\|_{L}\right)$ and fix an integer $k \in[0, L]$. Let $g=\sum_{i=0}^{k} \delta_{0}^{i}$ and 


$$
W_{1}=\left\{F \in X^{* *} \mid F(g)=k+1,\|F\|=1\right\} .
$$

Then there exists $F \in W_{1}$ such that

$$
F\left(u^{k}\right) \geq 0
$$

for any Borel measure $u$ on $[0,1]$, where $u^{k}$ is defined by (22). Moreover $F\left(m_{t}^{k}\right)=0$ for any $t \in[0,1]$, where

$$
m_{t}^{k}(f)=\int_{[0, t]} f^{(k)}(t) d m(t)
$$

and $m$ is the Lebesgue measure on $[0,1]$.

$$
\begin{gathered}
\text { Proof. Let } Z=\left(C^{L+1}[0,1],\|\cdot\|_{L+1}\right) . \text { Set } g=\sum_{i=0}^{k+1} \delta_{0}^{i} \text { and } \\
W_{2}=\left\{F \in Z^{* *} \mid F(g)=k+2,\|F\|=1\right\} .
\end{gathered}
$$

By Corollary $4.1, W_{2} \neq \emptyset$. Take any $G \in W_{2}$. Since $W_{2} \subset W$, by Theorem 4.3 we have $G\left(u^{k}\right) \geq 0$ for any Borel measure $u$, where $u^{k}$ is the functional on $Z$ defined by $(22)$. By the Goldstine theorem applied to $B\left(Z^{* *}\right)$, there exists a net $\left\{f_{\beta}\right\} \subset Z$ with $\left\|f_{\beta}\right\| \leq 1$ for any $\beta$ such that $f_{\beta} \rightarrow G$ weak* in $Z^{* *}$. Since $Z \subset X$ (as sets), we have $\left\{f_{\beta}\right\} \subset X$. Moreover, each $f_{\beta}$ has norm one in $X$, since its norm in $Z$ is at most one. By the Banach-Alaoglu theorem applied to $B\left(X^{* *}\right)$ the set $\left\{f_{\beta}\right\}$ has an accumulation point $F \in B\left(X^{* *}\right)$. Since $G \in W_{2}$,

$$
1=G\left(\delta_{0}^{i}\right)=\lim _{\beta} f_{\beta}^{(i)} \quad \text { for } i=0, \ldots, k+1 .
$$

Hence obviously $F\left(\delta_{0}^{i}\right)=1$ for $i=0, \ldots, k$. Since $\|F\| \leq 1$, we have $F \in W_{1}$. Moreover by Theorem 4.3, for any Borel measure $u$ on $[0,1]$,

$$
F\left(u^{k}\right)=\lim _{\beta} \int_{[0,1]} f_{\beta}^{(k)}(t) d u(t)=G\left(u^{k}\right) \geq 0,
$$

as desired. In particular, $F\left(m_{t}^{k}\right) \geq 0$ for any $t \in[0,1]$. By the fundamental theorem of calculus, for any $f \in X$,

$$
m_{t}^{k}(f)=\int_{[0, t]} f^{(k)}(t) d m(t)=f^{(k-1)}(t)-f^{(k-1)}(0) .
$$

Since $F \in W_{1}$,

$$
0 \leq F\left(m_{t}^{k}\right)=F\left(\delta_{t}^{k-1}-\delta_{0}^{k-1}\right)=F\left(\delta_{t}^{k-1}\right)-1 .
$$

Hence $F\left(\delta_{t}^{k-1}\right) \geq 1$. Since $\|F\|=1$,

$$
F\left(\delta_{t}^{k-1}\right)=1 .
$$

Consequently, $F\left(m_{t}^{k}\right)=0$, which completes the proof. 


\section{Proofs of minimality}

Proof of Theorem 2.3. The fact that $P_{0, n}=\sum_{i=0}^{n} u_{i} \otimes v_{i}$ is a projection follows from the definition of $u_{i}$ and $v_{i}$; it is easy to check that $\left\langle v_{i}, u_{j}\right\rangle=0$ unless $i=j$, in which case the result is 1 .

The verification that $P_{0, n}$ preserves the multi-convex shape described by $\boldsymbol{\sigma}$ (i.e., $P_{0, n} S_{\boldsymbol{\sigma}} \subset S_{\boldsymbol{\sigma}}$ ) is a direct calculation. Let $f \in S_{\boldsymbol{\sigma}}, t \in[0,1], j \leq M$ an integer and consider

$$
\begin{aligned}
\left\langle P_{0, n} f, \delta_{t}^{j}\right\rangle & =\left\langle\sum_{k=0}^{n-1} f^{(k)}(0) v_{k}+f^{(L-1)}(1) v_{n}, \delta_{t}^{j}\right\rangle \\
& =\sum_{k=j}^{n-1} f^{(k)}(0) v_{k}^{(j)}(t)+f^{(L-1)}(1) v_{n}^{(j)}(t) \geq 0
\end{aligned}
$$

since every term in the sum is nonnegative. Thus $P_{0, n} \in \mathcal{P}_{S_{\boldsymbol{\sigma}}}\left(X, \Pi_{n}\right)$.

To verify (3) of Theorem 2.3, note that, from (2), we have $\left\|P_{0, n}\right\| \leq$ $\sum_{k=0}^{n-1} 1 / k$ !. If $L=n-1$ then Lemma 4.4 yields $\left\|P_{0, n}\right\|=\sum_{k=0}^{n-1} 1 / k$ !. If $L \geq n$ Theorem 4.4 guarantees the existence of $F \in B\left(X^{* *}\right)$ such that $F\left(\delta_{0}^{i}\right)=1$ for $i=0, \ldots, n-1$ and $F(m)=0$, where $m$ denotes the Lebesgue measure. But we see from the proof of Theorem 4.4 that $F$ vanishing on $m$ implies $F\left(\delta_{1}^{n-1}\right)=1$, which implies $\left\|P_{0, n}\right\| \geq \sum_{k=0}^{n-1} 1 / k$ ! and thus (3) follows.

To show $P_{0, n}$ has minimal norm in $\mathcal{P}_{S_{\boldsymbol{\sigma}}}\left(X, \Pi_{n}\right)$, we consider two cases: $M=n-1$ and $M=n$. We handle the $M=n-1$ case first, using the following uniqueness argument.

We begin with a corollary given in [9]; it describes how the functionals that define a projection must be chosen in order for the projection to preserve shape.

Corollary 5.1 (see [9]). Suppose $P \in \mathcal{P}_{S}$. If $S_{\left.\right|_{V}}^{*}$ is $k$-dimensional then there exists a basis $\boldsymbol{v}=\left(v_{1}, \ldots, v_{n}\right)^{T}$ for $V$ such that whenever $P=\boldsymbol{u} \otimes \boldsymbol{v} \in$ $\mathcal{P}_{S}$, where $\boldsymbol{u}=\left(u_{1}, \ldots, u_{n}\right) \in\left(X^{*}\right)^{n}$, we have $u_{i} \in S^{*}$ for $i=n-k+1, \ldots, n$. Moreover, each such $u_{i}$ restricts to a distinct extreme ray of $S_{\left.\right|_{V}}^{*}$.

To utilize this result, we note that the proof of Theorem 2.1 demonstrates that the simplicial cone $S_{\left.\right|_{V}}^{*}$ has easily described extreme rays; they are generated (via nonnegative scalar multiplication) by

$$
\left\{\left(\delta_{0}\right)_{\left.\right|_{V}},\left(\delta_{0}^{1}\right)_{\left.\right|_{V}}, \ldots,\left(\delta_{0}^{n-1}\right)_{\left.\right|_{V}},\left(\delta_{1}^{n-1}\right)_{\left.\right|_{V}}\right\} .
$$

Thus, by Corollary 5.1, every projection $P=\sum_{i=0}^{n} u_{i} \otimes \widehat{v}_{i} \in \mathcal{P}_{S \boldsymbol{\sigma}}\left(X, \Pi_{n}\right)$ must be such that $u_{i} \in S^{*}$ and $\left(u_{i}\right)_{\left.\right|_{V}}=\left(\delta_{0}^{j}\right)_{\left.\right|_{V}}$ for some $j$ or $\left(u_{i}\right)_{\left.\right|_{V}}=$ $\left(\delta_{1}^{n-1}\right)_{\left.\right|_{V}}$. However, it is easy to check that for every $j$ there exists a unique element of $S^{*}$ whose restriction to $V$ is $\left(\delta_{0}^{j}\right)_{\left.\right|_{V}}$, namely $\delta_{0}^{j}$. Similarly, $\delta_{1}^{n-1}$ 
is the unique element of $S^{*}$ with restriction to $V$ given by $\left(\delta_{1}^{n-1}\right)_{\left.\right|_{V}}$. Consequently, (2) implies that $P_{0, n}$ is the unique element of $\mathcal{P}_{S_{\boldsymbol{\sigma}}}\left(X, \Pi_{n}\right)$ and therefore of minimal norm.

We now consider the case $M=n$. Unlike the previous case, the projection $P_{0, n}$ is not unique in $\mathcal{P}_{S_{\boldsymbol{\sigma}}}\left(X, \Pi_{n}\right)$; indeed, consider $P_{0, n}$ written in the following way:

$$
P_{0, n}=\delta_{0} \otimes 1+\delta_{0}^{1} \otimes \frac{x}{1 !}+\cdots+\delta_{0}^{n-1} \otimes \frac{x^{n-1}}{(n-1) !}+\left(\delta_{1}^{n-1}-\delta_{0}^{n-1}\right) \otimes \frac{x^{n}}{n !} .
$$

Replacing the functional $\delta_{1}^{n-1}-\delta_{0}^{n-1}$ with (a positive scalar multiple of) any nonzero element from the weak* closure of $\operatorname{cone}\left\{\delta_{t}^{n}\right\}_{t \in[0,1]}$ will result in an element of $\mathcal{P}_{S \boldsymbol{\sigma}}\left(X, \Pi_{n}\right)$. In fact, by Corollary 5.1 every element of $\mathcal{P}_{S \boldsymbol{\sigma}}\left(X, \Pi_{n}\right)$ can be constructed in this way. And that is why we are unable to appeal to the standard theory of minimal projections (described for example in [9]) which relies on best approximations from a linear space (and not from a cone). Therefore we proceed in the following way: we show that replacing $\delta_{1}^{n-1}-\delta_{0}^{n-1}$ in $P_{0, n}$ with any other allowable functional from $S^{*}$ results in an element of $\mathcal{P}_{S \boldsymbol{\sigma}}\left(X, \Pi_{n}\right)$ with norm at least as large as $\left\|P_{0, n}\right\|$. The following gives the form of an element from $\mathcal{P}_{S \boldsymbol{\sigma}}\left(X, \Pi_{n}\right)$ in the $M=n$ case.

Lemma 5.1. Let $Q \in \mathcal{P}_{S \boldsymbol{\sigma}}\left(X, \Pi_{n}\right)$. Then there exists $u \in X^{*}$ such that

$$
Q=\delta_{0} \otimes 1+\delta_{0}^{1} \otimes \frac{x}{1 !}+\cdots+\delta_{0}^{n-1} \otimes \frac{x^{n-1}}{(n-1) !}+u \otimes \frac{x^{n}}{n !} .
$$

Moreover, there exists a probability Borel measure $\mu$ such that for every $f \in X$ we have

$$
u(f)=\int_{0}^{1} f^{(n)}(t) d \mu(t) .
$$

Proof. Fix the basis $\left\{1, x, x / 2 !, \ldots, x^{n} / n\right.$ ! $\}$; then Corollary 5.1 guarantees the above representation of $Q$ and implies that $u \in S^{*} \subset X^{*}$. Furthermore $E\left(S^{*}\right)$, the union of the extreme rays of $S^{*}$, is (strictly) contained in the union of the rays

$$
\left\{\left[\delta_{t}^{j}\right]^{+} \mid t \in[0,1], j=0, \ldots, n\right\} .
$$

Let

$$
C=\overline{\mathrm{co}}\left(E\left(S^{*}\right) \cap S\left(X^{*}\right)\right)
$$

where the closure is taken with respect to the weak* topology. Note that $\operatorname{ext}(C) \subset\left\{\delta_{t}^{j} \mid t \in[0,1], j=0, \ldots, n\right\}$. Then by Proposition 2 from [31], for every nonzero $\phi \in S^{*}$, there exists a positive scalar $c$ and a probability Borel measure $\mu$ supported on $\operatorname{ext}(C)$ such that $\mu$ represents $c \phi$ (in the sense of 
Choquet), i.e.,

$$
c \phi(f)=\int_{\operatorname{ext}(C)} f(\delta) d \mu(\delta)
$$

for every $f \in X$. Consider now our $u$ above; the fact that $\left\langle x^{i}, u\right\rangle=0$ for every $i=0, \ldots, n-1$ implies that a representing measure $\mu$ for $u$ cannot have any positive support on the set of extreme points of $C$ of the form $\left\{\delta_{t}^{j} \mid t \in[0,1], j=0, \ldots, n-1\right\}$. And thus the representation in (27) is the only choice.

Now from Theorem 4.4, we have the existence of an $F \in B\left(X^{* *}\right)$ such that $F\left(\delta_{0}^{i}\right)=1$ for $i=0, \ldots, n-1$ and $F(u) \geq 0$. Therefore $\|Q\| \geq$ $\sum_{k=0}^{n-1} 1 / k$ !, which implies $P_{0, n}$ is of minimal norm in the $M=n$ case. This completes the proof of Theorem 2.3.

REMARK 5.1. Let $X=\left(C^{L}[0,1],\|\cdot\|_{2, L}\right)$, where

$$
\|f\|_{2, L}=\max \left\{\max _{j=0, \ldots, L-1}\left\{\left|f^{(j)}(0)\right|,\left|f^{(j)}(1)\right|\right\},\left\|f^{(L)}\right\|_{\infty}\right\} .
$$

Note that $\|\cdot\|_{L}$ and $\|\cdot\|_{2, L}$ are equivalent since

$$
(2 / 3)^{L}\|\cdot\|_{L} \leq\|\cdot\|_{2, L} \leq\|\cdot\|_{L} .
$$

Hence $P_{0, n} \in \mathcal{P}_{S_{\boldsymbol{\sigma}}}\left(X, \Pi_{n}\right)$. Moreover, an argument identical to the above shows $P_{0, n}$ is minimal in $\mathcal{P}_{S \boldsymbol{\sigma}}\left(X, \Pi_{n}\right)$ (for either $M=n-1$ or $M=n$ ) and (by Lemma 4.4 and Theorem 4.4) $\left\|P_{0, n}\right\|=\sum_{k=0}^{n-1} 1 / k$ !.

Proof of Theorem 2.4. To simplify notation, let $P$ denote the operator $P_{m+1, n+1}$ defined in (4). Also, for a positive integer $k$, we denote the Banach space $C^{k}[0,1]$ simply by $C^{k}$.

We begin by verifying that $P$ is a projection onto $\left[1, W_{0}, W_{1}, \ldots, W_{n}\right]$. Let $k(x)$ denote a (nonzero) constant function and note that

$$
(P k)(x)=(k(0)+k(1)) / 2=k(x)
$$

since $k^{\prime} \equiv 0$; thus $(P 1)(x)=1$. Moreover, using the fact that $P_{m, n}$ is a projection onto $Y$, for each integer $j \in[0, n]$ we have

$$
\left(P W_{j}\right)(x)=\left(W_{j}(0)+W_{j}(1)\right) / 2+\int_{0}^{x} w_{j}(t) d t-W_{j}(1) / 2=W_{j}(x)
$$

since $W_{j}(0)=0$. Thus $P$ is a projection onto $\left[1, W_{0}, W_{1}, \ldots, W_{n}\right]$.

Note the following (derivative) relationships between the projections $P$ and $P_{m, n}$ : for any $f \in C^{L+1}$, integer $j \in[1, L+1]$ and $x \in[0,1]$ we have

$$
(P f)^{j}(x)=\left(P_{m, n} f^{\prime}\right)^{(j-1)}(x) .
$$

To see that $P$ preserves shape, let $f \in S_{\widehat{\sigma}} \subset C^{L+1}$ and fix an integer $j \in[m+1, M+1]$. Then (28) implies $(P f)^{j}(x) \geq 0$ since $f^{\prime} \in S_{\boldsymbol{\sigma}} \subset C^{L}$ and 
$P_{m, n} \in \mathcal{P}_{S_{\boldsymbol{\sigma}}}\left(C^{L}, W\right)$. Hence

$$
P \in \mathcal{P}_{S_{\widehat{\boldsymbol{\sigma}}}}\left(C^{L+1},\left[1, W_{0}, W_{1}, \ldots, W_{n}\right]\right) .
$$

We now verify (5). Let $A=\left\{f^{\prime} \mid f \in B\left(C^{L+1}\right)\right\}$. We claim

$$
A=B\left(C^{L}\right) .
$$

Clearly $A \subset B\left(C^{L}\right)$. Let $g \in B\left(C^{L}\right)$ and define $f(x)=\int_{0}^{x} g(t) d t$. Obviously $f \in C^{L+1}$, since

$$
f^{(j)}(x)=g^{(j-1)}(x) \quad \text { for } j=1, \ldots, L+1 .
$$

Furthermore, from (30) it follows that $\left\|f^{(j)}\right\|_{\infty} \leq 1$ for $j=1, \ldots, L+1$. Finally, using the definition of $f$ we have $\|f\|_{\infty} \leq\|g\|_{\infty} \leq 1$ and thus $f \in B\left(C^{L+1}\right)$. This establishes our claim in (29) since $g=f^{\prime}$.

We are now ready to compare $\|P\|$ and $\left\|P_{m, n}\right\|$. Recall that

$$
\begin{aligned}
\|P\|=\sup _{f \in B\left(C^{L+1}\right)}\|P f\| & =\sup _{f \in B\left(C^{L+1}\right)} \max _{j=0, \ldots, L+1}\left\|(P f)^{(j)}\right\|_{\infty} \\
& =\max _{j=0, \ldots, L+1} \sup _{f \in B\left(C^{L+1}\right)}\left\|(P f)^{(j)}\right\|_{\infty} .
\end{aligned}
$$

Consider first the case $j \geq 1$; then

$$
\begin{aligned}
\sup _{f \in B\left(C^{L+1}\right)}\left\|(P f)^{(j)}\right\|_{\infty} & =\sup _{f \in B\left(C^{L+1}\right)} \sup _{x \in[0,1]}\left|(P f)^{(j)}(x)\right| \\
& =\sup _{f \in B\left(C^{L+1}\right)} \sup _{x \in[0,1]}\left|\left(P_{m, n} f^{\prime}\right)^{(j-1)}(x)\right| \quad \text { by }(30) \\
& =\sup _{f \in B\left(C^{L+1}\right)}\left\|\left(P_{m, n} f^{\prime}\right)^{(j-1)}\right\|_{\infty} \\
& =\sup _{f \in A}\left\|\left(P_{m, n} f\right)^{(j-1)}\right\|_{\infty} \\
& =\sup _{f \in B\left(C^{L}\right)}\left\|\left(P_{m, n} f\right)^{(j-1)}\right\|_{\infty} \quad \text { by }(29) .
\end{aligned}
$$

Consequently, we have

$$
\begin{aligned}
\max _{j=1, \ldots, M+1} \sup _{f \in B\left(C^{L+1}\right)}\left\|(P f)^{(j)}\right\|_{\infty} & =\max _{k=0, \ldots, M} \sup _{f \in B\left(C^{L}\right)}\left\|\left(P_{m, n} f\right)^{(k)}\right\|_{\infty} \\
& =\left\|P_{m, n}\right\| .
\end{aligned}
$$

To finish the comparison, we must check the $j=0$ case. Recalling the form of $P\left(=P_{m+1, n+1}\right)$ given in (4), we find

$$
\begin{aligned}
\sup _{f \in B\left(C^{L+1}\right)} & \|P f\|_{\infty} \\
& \leq 1+\sup _{f \in B\left(C^{L+1}\right)}\left\|\int_{0}^{x}\left(P_{m, n} f^{\prime}\right)(t) d t-\frac{1}{2} \int_{0}^{1}\left(P_{m, n} f^{\prime}\right)(t) d t\right\| .
\end{aligned}
$$


However, the right-hand side of (32) becomes

$$
\begin{aligned}
1+\sup _{f \in B\left(C^{L+1}\right)} & \left\|\frac{1}{2} \int_{0}^{x}\left(P_{m, n} f^{\prime}\right)(t) d t-\frac{1}{2}\left(\int_{0}^{1}\left(P_{m, n} f^{\prime}\right)(t) d t-\int_{0}^{x}\left(P_{m, n} f^{\prime}\right)(t) d t\right)\right\|_{\infty} \\
& =1+\frac{1}{2} \sup _{f \in B\left(C^{L+1}\right)}\left\|\int_{0}^{x}\left(P_{m, n} f^{\prime}\right)(t) d t-\int_{x}^{1}\left(P_{m, n} f^{\prime}\right)(t) d t\right\|_{\infty} \\
& =1+\frac{1}{2} \sup _{f \in B\left(C^{L+1}\right)} \sup _{x \in[0,1]}\left|\int_{0}^{x}\left(P_{m, n} f^{\prime}\right)(t) d t-\int_{x}^{1}\left(P_{m, n} f^{\prime}\right)(t) d t\right| \\
& \leq 1+\frac{1}{2} \sup _{f \in B\left(C^{L+1}\right)} \int_{0}^{1}\left|\left(P_{m, n} f^{\prime}\right)(t)\right| d t \\
& =1+\frac{1}{2} \sup _{f \in B\left(C^{L}\right)} \int_{0}^{1}\left|\left(P_{m, n} f\right)(t)\right| d t \quad \text { by }(29) \\
& \leq 1+\frac{1}{2}\left\|P_{m, n}\right\| .
\end{aligned}
$$

Thus

$$
\sup _{f \in B\left(C^{L+1}\right)}\|P f\|_{\infty} \leq 1+\frac{1}{2}\left\|P_{m, n}\right\| \leq\left\|P_{m, n}\right\|
$$

since, by assumption, $\left\|P_{m, n}\right\| \geq 2$. This result, in combination with (31), establishes (5) and completes the proof of Theorem 2.4.

REMARK 5.2. This proof demonstrates that when $C^{L}[0,1]$ is normed by $\|\cdot\|_{L}$, the construction given in (4) is (operator) norm-preserving. It is a straightforward verification that this proof can be repeated when $\|\cdot\|_{L}$ is replaced by $\|\cdot\|_{2, L}$ and thus we have norm preservation in this case as well.

Proof of Theorem 2.5. We begin by verifying this theorem in the $(L+k)$ norm case. For simplicity, let $X_{L+k}=\left(C^{L+k}[0,1],\|\cdot\|_{L+k}\right)$. From our assumption on the construction of $P_{k, n+k}$ and Theorem 2.4 we have $P_{k, n+k} \in$ $\mathcal{P}_{S_{\boldsymbol{\sigma}}}\left(X_{L+k}, \Pi_{n+k}\right)$ and

$$
\left\|P_{k, n+k}\right\|=\left\|P_{0, n}\right\|
$$

In fact, we can say more: a straightforward generalization of (29) gives

$$
\left\{f^{(k)} \mid f \in B\left(X_{L+k}\right)\right\}=B\left(X_{L}\right)
$$

and so

$$
\begin{aligned}
\left\|P_{0, n}\right\| & =\sup _{f \in B\left(X_{L}\right)}\left\|P_{0, n} f\right\|_{\infty}=\sup _{f \in B\left(X_{L+k}\right)}\left\|P_{0, n}\left(f^{(k)}\right)\right\|_{\infty} \\
& \left.=\sup _{f \in B\left(X_{L+k}\right)} \|\left(P_{k, n+k} f\right)^{(k)}\right) \|_{\infty} .
\end{aligned}
$$


This result, together with (33), yields

$$
\left.\left\|P_{k, n+k}\right\|=\sup _{f \in B\left(X_{L+k}\right)} \|\left(P_{k, n+k} f\right)^{(k)}\right) \|_{\infty} .
$$

Let $Q \in \mathcal{P}_{S \boldsymbol{\sigma}}\left(X_{L+k}, \Pi_{n+k}\right)$. Recall $M=\max _{\sigma_{i}=1} i$. Then by Corollary 5.1 there exists a basis $\left\{\widehat{v}_{0}, \widehat{v}_{1}, \ldots, \widehat{v}_{n+k}\right\}$ for $\Pi_{n+k}$ such that $Q$ may be represented as

$$
Q=\sum_{i=0}^{k-1} \phi_{i} \otimes \widehat{v}_{i}+\sum_{i=k}^{n+k-1} \delta_{0}^{i} \otimes \widehat{v}_{i}+\Delta \otimes \widehat{v}_{n+k}
$$

where, in the case $M=n+k-1, \Delta=\delta_{1}^{n+k-1}$ and otherwise (for $M=n+k$ ) $\Delta$ is any nonzero element of the weak* closure of the cone generated by the set $\left\{\delta_{t}^{n+k}\right\}_{t \in[0,1]}$.

We claim that for $i=0, \ldots, k-1$, the degree of $\widehat{v}_{i}$ is strictly less than $k$. To the contrary, suppose for some $i$ we have $k \leq \operatorname{deg}\left(\widehat{v}_{i}\right) \leq n+k$. If $\operatorname{deg}\left(\widehat{v}_{i}\right)<n+k$ then $\left\langle\widehat{v}_{i}, \delta_{0}^{\operatorname{deg}\left(\widehat{v}_{i}\right)}\right\rangle \neq 0$, which is a contradiction; a similar conclusion is obtained if $\operatorname{deg}\left(\widehat{v}_{i}\right)=n+k$ since $\left\langle\widehat{v}_{i}, \Delta\right\rangle \neq 0$. Thus $\operatorname{deg}\left(\widehat{v}_{i}\right)<k$ for $i=0, \ldots, k-1$.

For $i=k, \ldots, n+k$, write $\widehat{v}_{i}=a_{i} v_{i}+p_{i}$ where $a_{i} \in \mathbb{R}, v_{i}$ is as in Theorem 2.3 , and $p_{i} \in \Pi_{n+k}$. Using an orthogonality argument identical to that above (e.g., $\left\langle\widehat{v}_{i}, \delta_{0}^{j}\right\rangle=0$ whenever $i \neq j$ ), we conclude that $a_{i}=1$ and $\operatorname{deg}\left(p_{i}\right)<k$. Thus

$$
(Q f)^{(k)}=\left(P_{k, n+k} f\right)^{(k)}
$$

and so by (34) we find

$$
\|Q\| \geq \sup _{f \in B\left(X_{L+k}\right)}\left\|(Q f)^{(k)}\right\|_{\infty}=\sup _{f \in B\left(X_{L+k}\right)}\left\|\left(P_{k, n+k} f\right)^{(k)}\right\|_{\infty}=\left\|P_{k, n+k}\right\| .
$$

Therefore $P_{k, n+k}$ has minimal norm in $\mathcal{P}_{S_{\boldsymbol{\sigma}}}\left(X_{L+k}, \Pi_{n+k}\right)$.

Consider now the case $X_{2, L+k}=\left(C^{L+k}[0,1],\|\cdot\|_{2, L+k}\right)$. From our assumption on the construction of $P_{k, n+k}$ and Remarks 5.1 and 5.2 we know that $P_{k, n+k} \in \mathcal{P}_{S_{\boldsymbol{\sigma}}}\left(X_{2, L+k}, \Pi_{n+k}\right)$ and

$$
\left\|P_{k, n+k}\right\|_{2, L+k}=\left\|P_{0, n}\right\|_{2, L},
$$

where $\|Q\|_{2, L+k}$ denotes the operator norm of $Q$ defined on $X_{2, L+k}$. From Remark 5.1 the norm $\left\|P_{0, n}\right\|_{2, L}$ is minimal (in view of Theorem 2.3 and Remark 5.1) and therefore, from an argument identical to that above in the $L$-norm case, we conclude that $P_{k, n+k}$ is a minimal norm element from $\mathcal{P}_{S_{\boldsymbol{\sigma}}}\left(X_{2, L+k}, \Pi_{n+k}\right)$.

We now make the following observation: the operator

$$
P_{k, n+k}: X_{L+k}=\left(C^{L+k}[0,1],\|\cdot\|_{L+k}\right) \rightarrow Y=\left(\Pi_{n+k},\|\cdot\|_{2, L+k}\right)
$$

preserves the multi-convex shape $S_{\boldsymbol{\sigma}}$. From the form of $P_{k, n+k}$, it follows that the operator norm of $P_{k, n+k}: X_{L+k} \rightarrow Y$ is equal to the operator norm 
of $P_{k, n+k} \in \mathcal{P}_{S_{\boldsymbol{\sigma}}}\left(X_{2, L+k}, \Pi_{n+k}\right)$. Let $\left\|P_{k, n+k}\right\|$ denote this common value. Again using the form of $P_{k, n+k}$ we find

$$
\left\|P_{k, n+k}\right\|=\sup _{f \in B\left(X_{2, L+k}\right)} \sup _{t \in\{0,1\}}\left|\left(P_{k, n+k} f\right)^{(k)}(t)\right| .
$$

We claim that $P_{k, n+k}$ has minimal (operator) norm among all operators between $X_{L+k}$ and $Y$ preserving $S_{\boldsymbol{\sigma}}$. Indeed, let $Q$ be any such operator. By Remark 5.1 the norms $\|\cdot\|_{L+k}$ and $\|\cdot\|_{2, L+k}$ are equivalent and therefore we may consider $Q$ as an element of $\mathcal{P}_{S \sigma}\left(X_{L+k}, \Pi_{n+k}\right)$, i.e., a projection from $X_{L+k}$ onto $\Pi_{n+k}$ such that $Q S_{\boldsymbol{\sigma}} \subset S_{\boldsymbol{\sigma}}$. This implies that $Q: X_{L+k} \rightarrow Y$ has the form described in (35) and therefore we have (36). The minimality of $P_{k, n+k}: X_{L+k} \rightarrow Y$ follows since

$$
\begin{aligned}
\|Q\| & =\sup _{f \in B\left(X_{L+k}\right)}\|Q f\|_{2, L+k} \\
& \geq \sup _{f \in B\left(X_{L+k}\right)} \sup _{t \in\{0,1\}}\left|(Q f)^{(k)}(t)\right|=\sup _{f \in B\left(X_{L+k}\right)} \sup _{t \in\{0,1\}}\left|\left(P_{k, n+k} f\right)^{(k)}(t)\right| \\
& =\sup _{f \in B\left(X_{2, L+k}\right)} \sup _{t \in\{0,1\}}\left|\left(P_{k, n+k} f\right)^{(k)}(t)\right|=\left\|P_{k, n+k}\right\| .
\end{aligned}
$$

Finally, let $X=\left(C^{L+k}[0,1],\|\cdot\|\right)$ be such that

$$
\|\cdot\|_{2, L+k} \leq\|\cdot\| \leq\|\cdot\|_{L+k} .
$$

From the definitions of the $L$ - and $(2, L)$-norms, we have

$$
\left\|P_{k, n+k}\right\|_{2, L+k} \leq\left\|P_{k, n+k}\right\|_{X} \leq\left\|P_{k, n+k}\right\|_{L+k}
$$

where $\left\|P_{k, n+k}\right\|_{X}$ is the operator norm of $P_{k, n+k}$ defined on $X$. But from (33), (37) and Remark 5.1 we find

$$
\left\|P_{k, n+k}\right\|_{X}=\left\|P_{k, n+k}\right\|_{2, L+k}=\left\|P_{k, n+k}\right\|_{L+k} .
$$

As above, let $\left\|P_{k, n+k}\right\|$ denote this common value. To show that $P_{k, n+k}$ has minimal norm in $\mathcal{P}_{S \boldsymbol{\sigma}}\left(X, \Pi_{n+k}\right)$ let $Q \in \mathcal{P}_{S_{\boldsymbol{\sigma}}}\left(X, \Pi_{n+k}\right)$. Since $Q: X_{L+k} \rightarrow Y$ with $Q S_{\boldsymbol{\sigma}} \subset S_{\boldsymbol{\sigma}}$, we have

$$
\|Q\|_{X}=\sup _{f \in B(X)}\|Q f\| \geq \sup _{f \in B\left(X_{L+k}\right)}\|Q f\|_{2, L+k} \geq\left\|P_{k, n+k}\right\|
$$

\section{References}

[1] J. Blatter and E. W. Cheney, Minimal projections onto hyperplanes in sequence spaces, Ann. Mat. Pura Appl. 101 (1974), 215-227.

[2] H. F. Bohnenblust, Subspaces of $l_{p, n}$-spaces, Amer. J. Math. 63, (1941), 64-72.

[3] B. L. Chalmers and G. Lewicki, Symmetric subspaces of $l_{1}$ with large projection constants, Studia Math. 134 (1999), 119-133.

[4] -, -, Two-dimensional real symmetric spaces with maximal projection constants, Ann. Polon. Math. 72 (2000), 119-134. 
[5] B. L. Chalmers and G. Lewicki, Symmetric spaces with maximal projection constants, J. Funct. Anal. 200 (2003), 1-22.

[6] B. L. Chalmers and F. T. Metcalf, The determination of minimal projections and extensions in $L_{1}$, Trans. Amer. Math. Soc. 329 (1992), 289-305.

[7] - - - A characterization and equations for minimal projections and extensions, J. Operator Theory 32 (1994), 31-46.

[8] - - - Determination of a minimal projection from $C[-1,1]$ onto the quadratics, Numer. Funct. Anal. Optim. 11 (1990), 1-10.

[9] B. L. Chalmers, D. Mupasiri and M. P. Prophet, A characterization and equations for minimal shape-preserving projections, J. Approx. Theory 138 (2006), 184-196.

[10] - - - Existence of shape preserving A-action operators, Rocky Mountain J. Math. 28 (1998), 813-833.

[11] —, - Minimal shape preserving projections, Numer. Funct. Anal. Optim. 18 (1997), $507-520$.

[12] B. L. Chalmers, M. P. Prophet and J. Ribando, Simplicial cones and the existence of shape-preservation cyclic operators, Linear Algebra Appl. 375 (2003), 157-170.

[13] E. W. Cheney and C. Franchetti, Minimal projections in $L_{1}$-spaces, Duke Math. J. 43 (1976), 501-510.

[14] E. W. Cheney, C. R. Hobby, P. D. Morris, F. Schurer and D. E. Wulbert, On the minimal property of the Fourier projection, Trans. Amer. Math. Soc. 143 (1969), 249-258.

[15] E. W. Cheney and P. D. Morris, On the existence and characterization of minimal projections, J. Reine Angew. Math. 270 (1974), 61-76.

[16] S. D. Fisher, P. D. Morris and D. E. Wulbert, Unique minimality of Fourier projections, Trans. Amer. Math. Soc. 265, (1981), 235-246.

[17] C. Franchetti, Projections onto hyperplanes in Banach spaces, J. Approx. Theory 38 (1983), 319-333.

[18] J. R. Isbell and Z. Semadeni, Projection constants and spaces of continuous functions, Trans. Amer. Math. Soc. 107 (1963), 38-48.

[19] J. E. Jamison, A. Kamińska and G. Lewicki, One-complemented subspaces of Musielak-Orlicz sequence spaces, J. Approx. Theory 130 (2004), 1-37.

[20] H. König, Spaces with large projection constants, Israel J. Math. 50 (1985), 181-188.

[21] H. König and N. Tomczak-Jaegermann, Bounds for projection constants and 1summing norms, Trans. Amer. Math. Soc. 320 (1990), 799-823.

[22] —, - Norms of minimal projections, J. Funct. Anal. 119 (1994), 253-280.

[23] G. Lewicki, Kolmogorov's type criteria for spaces of compact operators, J. Approx. Theory 64 (1991), 181-202.

[24] -, Minimal projections onto two dimensional subspaces of $l_{\infty}^{(4)}$, ibid. 88 (1997), 92-108.

[25] —, Minimal extensions in tensor product spaces, ibid. 97 (1999), 366-383.

[26] -, On minimal projections in $l_{\infty}^{(n)}$, Monatsh. Math. 129 (2000), 119-131.

[27] G. Lewicki, G. Marino and P. Pietramala, Fourier-type minimal extensions in real $L_{1}$-spaces, Rocky Mountain J. Math. 30 (2000), 1025-1037.

[28] G. Lewicki and M. P. Prophet, Minimal shape preserving projections: generalizations and extensions, Numer. Funct. Anal. Optim. 27 (2006), 847-873.

[29] J. Lindenstrauss, On projections with norm one-an example, Proc. Amer. Math. Soc. 15 (1964), 403-406. 
[30] F. J. Muñoz-Delgado, V. Ramírez-González and D. Cárdenas-Morales, Qualitative Korovkin-type results on conservative approximation, J. Approx. Theory 94 (1998), 144-159.

[31] D. Mupasiri and M. P. Prophet, A note on the existence of shape-preserving projections, Rocky Mountain J. Math., to appear.

[32] W. Odyniec and G. Lewicki, Minimal Projections in Banach Spaces, Lecture Notes in Math. 1449, Springer, Berlin, 1990.

[33] K. C. Pan and B. Shekhtman, On minimal interpolating projections and trace duality, J. Approx. Theory 65 (1991), 216-230.

[34] M. P. Prophet, On j-convex preserving interpolation operators, ibid. 104 (2000), $77-89$.

[35] —, Codimension one minimal projections onto the quadratics, ibid. 85 (1996) 27-42.

[36] B. Randrianantoanina, Contractive projections in nonatomic function spaces, Proc. Amer. Math. Soc. 123 (1995), 1747-1750.

[37] -, One-complemented subspaces of real sequence spaces, Results Math. 33 (1998), 139-154.

[38] S. Rolewicz, On minimal projections of the space $L([0,1])$ on 1-codimensional subspace, Bull. Polish Acad. Sci. Math. 34 (1986), 151-153.

[39] L. Skrzypek, The uniqueness of norm-one projection in James-type spaces, J. Approx. Theory 100 (1997), 73-93.

[40] - Uniqueness of minimal projections in smooth matrix spaces, ibid. 107 (2000), 315-336.

[41] - Minimal projections in spaces of functions of $n$ variables, ibid. 123 (2003), 214231.

[42] D. E. Wulbert, Some complemented function spaces in $C(X)$, Pacific J. Math. 24 (1968), 589-602.

Department of Mathematics

Jagiellonian University

Reymonta 4

30-059 Kraków, Poland

E-mail: Grzegorz.Lewicki@im.uj.edu.pl
Department of Mathematics

University of Northern Iowa Cedar Falls, IA 50614-0506, U.S.A.

E-mail: prophet@math.uni.edu

Received April 25, 2005

Revised version November 2, 2006 